\title{
FORMATION OF LOW-MASS BLACK HOLE X-RAY TRANSIENTS
}

\author{
SIMON PORTEGIES ZWART \\ Astronomical Institute Anton Pannekoek, Amsterdam, The Netherlands \\ FRANK VERBUNT \\ Astronomical Institute Utrecht, The Netherlands \\ AND \\ ENE ERGMA \\ Tartu University, Tartu, Estonia
}

\section{Introduction}

We study the formation of low-mass X-ray binaries with a black hole as accreting object. The performed semi-analytic analysis reveals that the formation rate of black holes in low-mass X-ray binaries is about two orders of magnitude smaller than that of systems with a neutron star as accretor. This is contradicted by the six observed systems, which are all transients, which suggest that the majority of these systems has not been seen jet. The birthrate for both type of objects are expected to be similar (for reviews see Cowley 1992, Tanaka \& Lewin 1995).

Solution of this conundrum requires either that stars with masses less than $20 \mathrm{M}_{\odot}$ can evolve into black holes, or that stellar wind from a member of a binary is accompanied by a much larger loss of angular momentum than hitherto assumed.

The most popular formation scenario for low-mass X-ray binaries proposes a relatively wide binary with an extremely small mass ratio as the progenitor system (van den Heuvel 1983). The massive star evolves to fill its Roche lobe, engulfs its low-mass companion followed by a spiral-in. A close binary remains if the spiral-in ceases before the low-mass companion coalesces with the compact helium core of the primary. The helium core continues its evolution and may turn into a neutron star or a black hole. 


\section{The maximum initial separation for Roche-lobe contact}

In the standard scenario the formation of a low-mass X-ray binary starts with a high-mass primary $M_{\circ}$ and a low mass secondary star $\left(m_{\circ} \sim 1 \mathrm{M}_{\odot}\right)$ in a detached binary with semi-major axis $a_{\circ}$; the initial mass-ratio $m_{\circ} / M_{\circ}$ is consequently fairly small. For simplicity we assume that the initial orbit is circular. As the primary evolves and expands on the giant branch it might lose a considerable fraction of its mass in a stellar wind before the primary fills its Roche-lobe. This results in an expansion of the orbit according to the Jeans approximation of conservation of angular momentum (see van den Heuvel 1983): $a(M+m)=a_{\circ}\left(M_{\circ}+m_{\circ}\right)$. The initial orbital separation $a_{\circ}$ follows from the assumption that the primary with mass $M\left(<M_{\circ}\right)$ and radius $R\left(R \gg R_{\circ}\right)$ just fits the size of its Roche lobe (Eggleton 1983).

For each stellar evolution track in the tables of Schaller et al. (1992) we can now compute the maximum to the initial semi-major axis $a_{\max }$ for which the star fills its Roche lobe. Note that mass loss by the stellar wind might cause $a_{\max }$ to be reached at an earlier epoch than at the maximum radius of the primary (see Fig. 2 in Portegies Zwart et al. 1997).

\section{The minimum initial separation to survive mass transfer}

Roche-lobe contact to the small mass companion causes mass transfer to be highly unstable and a common-envelope phase cannot be avoided. A close binary remains after the spiral in provided that the primary's envelope is fully ejected before the secondary star coalesces with the compact core (with mass $M_{\mathrm{He}}=0.073 M_{\circ}^{1.42}$ ) of the primary. The minimum orbital separation after the spiral in is provided by the secondary with mass $m\left(\equiv m_{\circ}\right)$ and radius $r\left(\equiv r_{\circ}\right)$ to fill its Roche-lobe. The core of the primary is more massive than its companion and its size is smaller. The secondary therefore gives the strongest limit.

The reduction in the semi-major axis during the spiral-in can be computed by comparing the binding energy of the primary's envelope with the orbital binding-energy of the binary (Webbink 1984): $\Delta E_{\text {env }}=\alpha_{c e} \Delta E_{\text {orb }}$. Here $\alpha_{c e}$ gives the efficiency in which the energy generated by the friction between the secondary and the common envelope will blow off the envelope at the cost of orbital energy. The minimum semi-major axis $a_{\min }$ for which the binary survives spiral-in is computed accordingly.

From the orbital separation that corresponds barely to survival of the common-envelope we can compute the initial orbital separation by applying Jeans approximation for the phase of mass loss before Roche-lobe contact. The masses and radii of the primary, required for this study, are taken from stellar evolution tracks of Schaller et al. (1992). Figure 1 gives the minimum and maximum to the initial semi-major axes. There where the solid line is 


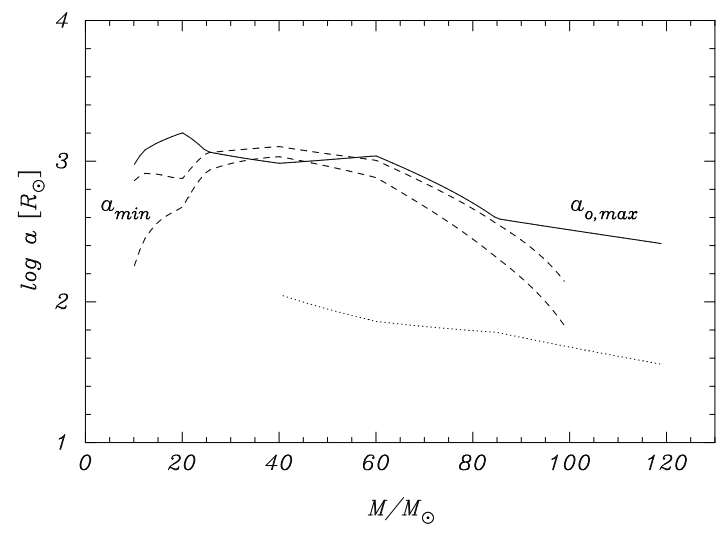

Figure 1. Lower limit to the initial semi-major axis at which the binary survives the spiral-in, as function of the initial mass of the primary, calculated with use of the evolutionary sequences by Schaller et al. (1992). The upper (lower) dashed line gives the limit determined from the condition that the secondary star (helium core) is smaller than its Roche-lobe, The solid line gives the upper limit at which the primary reaches its Roche lobe at its maximum radius. The secondary is assumed to be a $1 \mathrm{M}_{\odot}$ star. The dotted line indicates the initial semi-major axis of a binary in which a $1 \mathrm{M}_{\odot}$ secondary can fill its Roche lobe after the primary has lost its entire envelope without ever having filled its Roche lobe

above the dashed line primaries are able to fill their Roche-lobe and survive the spiral-in. The small surface area enclosed by the solid line $\left(a_{\circ, \max }\right)$ and the upper dashed line $\left(a_{\min }\right)$ gives the allotted region for the formation of a low-mass X-ray binary.

\subsection{RELATIVE BIRTHRATE}

The formation rate of binaries that reach Roche-lobe contact and survive the spiral-in can be estimated by computing the birth rate of binaries with suitable initial parameters; primary mass, mass ratio and semi-major axis. For simplicity we neglect the eccentricity. For the initial mass function for the primary we use a Salpeter function (Salpeter 1964). The initial semi-major axis distribution is taken flat in $\log a$ and the initial mass-ratio distribution is taken uniform between 0 and 1 .

Primaries with an initial mass between $10 \mathrm{M}_{\odot}$ and $40 \mathrm{M}_{\odot}$ are assumed to leave a neutron star after the supernova, progenitors with a mass between $40 \mathrm{M}_{\odot}$ and $100 \mathrm{M}_{\odot}$ form black holes. The secondary star is assumed to have a mass between $0.85 \mathrm{M}_{\odot}$ and $1.15 \mathrm{M}_{\odot}$. The minima and maxima for the initial semi-major axis are computed as described in the previous section. We integrate the initial distribution functions for the primary mass, the mass ratio and the semi-major axis between the appropriate mass limits and the corresponding limits for the semi-major axis $a_{\min }$ and $a_{\circ, \max }$ 


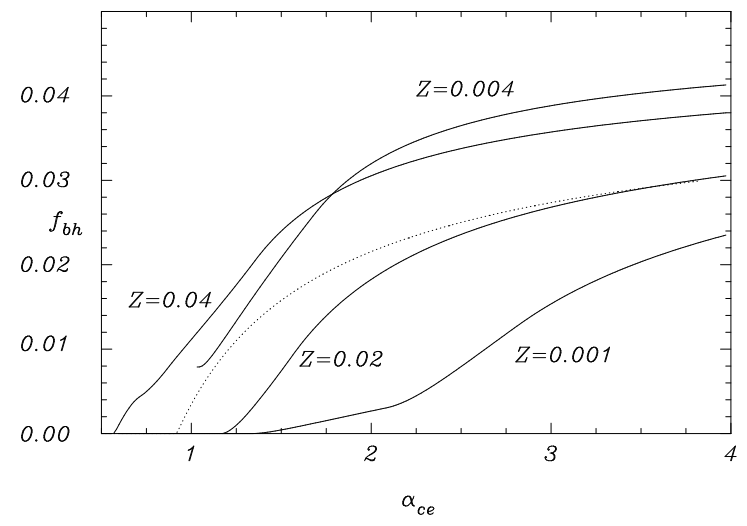

Figure 2. The binaries with a black hole as a fraction of the neutron-star binaries (X-axis) as a function of the common-envelope parameter $\alpha_{c e}$. The different lines give the result for stellar evolution models with different metalicities: $Z=0.001$ (Schaller et al. 1992), $Z=0.004$ (Charbonnel et al. 1993), $Z=0.02$ (solid: Maeder \& Meynet 1988, dotted: Schaller et al. 1992) and $Z=0.04$ (Schaerer et al. 1993).

(see Fig. 1): With the proper normalization for the initial mass function and a binary fraction of $50 \%$ the galactic formation rate of binaries that reach Roche-lobe contact and survive the spiral-in is $2.2 \cdot 10^{-6} \mathrm{yr}^{-1}$ for the binaries with a neutron star (which is comparable to the estimates from observations) and $9.6 \cdot 10^{-9} \mathrm{yr}^{-1}$ for the black-hole binaries (see Portegies Zwart et al. 1997). The formation rate of low-mass X-ray binaries with a black hole is about $1 \%$ of the formation rate of low-mass X-ray binaries with a neutron star, whereas the observations indicate equal formation rates for these two types of binaries. Figure 2 gives the relative birthrates of lowmass X-ray binaries with a black hole relative to those with a neutron star for various metalicities ranging from $Z=0.001$ to $Z=0.04$ as a function of the common envelope efficiency parameter $\alpha_{c e}$.

\section{Conclusions}

Our computations predict a formation rate for low mass X-ray binaries with a black hole which is much smaller than the value derived from the observed numbers and estimated X-ray lifetime. The discrepancy between theoretical and observed formation rates cannot be solved by invoking different metalicities for the progenitor systems, nor by assuming different efficiencies for the envelope rejection during spiral-in.

Black-hole binaries can be produced in larger numbers only if we assume that stars with initial masses less than $\sim 20 \mathrm{M}_{\odot}$ collapse in to black holes; or alternatively if it is assumed that the angular momentum loss caused by the stellar wind is so high that the binary orbit shrinks. 


\section{Acknowledgements}

This work was supported in part by the Netherlands Organization for Scientific Research (NWO) under grant PGS 13-4109 and by the Leids Kerkhoven Boscha Fonds. This investigation is supported by NWO under Pioneer grand PGS 78-277 to F. Verbunt and by Spinoza grant 08-0 to E. P. J. van den Heuvel.

\section{References}

Charbonnel, C., Meynet, G., Maeder, A. S., Schaller, G., Schaerer, D. 1993, A\&AS, 101, 415

Cowley, A. 1992, ARA\&A, 30, 287

Maeder, A., Meynet, G. 1989, A\&A, 210, 155

Salpeter, E. E. 1964, ApJ, 140, 796

Schaerer, D., Charbonnel, C., Meynet, G., Maeder, A. S., Schaller, G. 1993, A\&AS, 102, 339

Schaller, G., Schaerer, D., Meynet, G., Maeder, A. S. 1992, A\&AS, 96, 269

Portegies Zwart, S. F., Verbunt, F., Ergma, E., 1997, A\&A 321, 207

Tanaka, Y., Lewin, W. H. G. 1995, in W. H. G. e. a. Lewin (ed.), X-ray binaries, Cambridge University Press, 536

van den Heuvel, E. 1983, in W. Lewin, E. van den Heuvel (eds.), Accretion-driven stellar X-ray sources, Cambridge U.P., Cambridge, p. 303

van den Heuvel, E. P. J., Habets, G. 1984, Nat, 309, 598

Webbink, R. F. 1984, ApJ, 277, 355 